\title{
¿Robots o programación? El concepto de Pensamiento Computacional y los futuros maestros
}

\section{Robotics or Coding? The Concept of Computational Thinking in Pre-service Teachers}

Juan González Martínez, Meritxell Estebanell Minguell, Marta Peracaula Bosch

UdiGitalEdu. Universitat de Girona. Serra Húnter Fellow

\{juan.gonzalez, meritxell.estebanell, marta.peracaula\}@ udg.edu

https://orcid. org/0000-0002-9175-6369

https://orcid.org/0000-0002-6576-3207

https://orcid.org/0000-0003-0871-6583

\section{Resumen}

\begin{abstract}
El Pensamiento Computacional, por medio de la robótica o de la programación, está siendo incluido en el currículum en todo el mundo. No en vano, se considera una de las competencias necesarias para el siglo XXI. Sin embargo, los docentes a menudo no han sido formados para enseñar Pensamiento Computacional y ni siquiera saben exactamente qué es. Por ello, intentamos conocer cuál es el concepto de Pensamiento Computacional por parte de los maestros en formación por medio de una metodología mixta, que nos permite detectar importantes faltas de conocimiento y errores en relación con el concepto. Sin embargo, por otro lado, se diagnostican buenas actitudes iniciales hacia el Pensamiento Computacional, que pueden permitir que la formación específica recibida en este ámbito sea transferible a contextos futuros de práctica docente.
\end{abstract}

\section{Palabras Clave}

Pensamiento Computacional; Formación de maestros; $\mathrm{TIC}$

\begin{abstract}
Computational Thinking, through robotics or coding experiences, is being included in the school curriculum around the world. It is considered one of the necessary skills for surviving in the 21st century. However, teachers have often not been trained to teach Computational Thinking at school and do not even know exactly what it is. Therefore, we try to understand the concept of Computational Thinking by teachers in training through a mixed methodology, which allows us to detect important knowledge and errors in relation to this concept. However, on the other hand, good initial attitudes toward Computational Thinking are diagnosed, which can allow the specific training received in this field to be transferable to future contexts of teaching practice.
\end{abstract}

Keywords

Computational Thinking; Pre-service Teachers; ICTS

\section{El Pensamiento Computacional en educación}

En los últimos años, la sociedad ha asistido a una auténtica revolución por lo que respecta a los fundamentos que deben guiar la educación que se ofrece a los nuevos ciudadanos. La Sociedad del Conocimiento está en continuo cambio, no solo en relación con los recursos de que se dispone, sino también en relación con cómo aprendemos o qué debemos aprender (Cela-Ranilla, Esteve, Esteve Mon, González Martínez, \& Gisbert Cervera, 2017). Es en esa clave bajo la cual se están replanteando 
cuáles son las necesidades de formación de las personas del siglo XXI, en un contexto caracterizado por la denominada VUCA (por las siglas inglesas derivadas de volatilidad, incertidumbre, complejidad y ambigüedad) (Acevedo Borrega, 2016). El futuro nos deparará nuevos retos que actualmente no somos capaces siquiera de imaginar, y a los que tendremos que hacer frente con conocimientos que acaso aún estén por descubrir. Por ello, es imprescindible que el sistema educativo forme ciudadanos competentes para afrontar dichas situaciones, cualesquiera que estas sean. En esas coordenadas, venimos hablando del Pensamiento Computacional (en adelante, PC) como una de las competencias que deben permitir a cualquier ciudadano, con independencia de su quehacer profesional, la gestión óptima de todas las situaciones. En efecto, ahí empieza la reflexión de Wing (2006), cuando destaca que el PC permitirá a los alumnos, como herramienta básica transversal al currículum, aprender de forma abstracta, algorítmica y lógica y, por ende, les preparará para resolver problemas complejos y abiertos.

Esta misma idea ha sido seguida por muchos autores, que identifican el PC como una de las destrezas básicas que debe adquirir el ciudadano del siglo XXI (Angeli et al., 2016; European Commission/ EACEA/Eurydice, 2012; Fluck et al., 2016), y cuyas potencialidades radican en desarrollar un único procedimiento eficaz para la resolución de problemas, empoderar a los individuos para la creación de soluciones propias (artefactos o procedimientos) y redefinir la relación entre el aprendiz y el resto de las disciplinas que aprende, más allá del propio PC (Fluck et al., 2016). Y, en la misma línea, Furber (2012) señala que el PC parte de la idea de reconocer aspectos de la computación en el mundo que nos rodea, así como aplicar herramientas y técnicas de la computación para entender y razonar acerca de los procesos tanto naturales como artificiales de nuestro contexto ordinario. Finalmente, por su parte, Grover y Pea (2013) focalizan la importancia del PC en siete aspectos, a saber: (1) la computación es una actividad humana; (2) la abstracción ayuda a reducir la información y (3) a focalizar en los aspectos esenciales; (4) Los procesos derivados del PC contribuyen a la creación de conocimiento; (5) programar es un proceso creativo; (6) el desarrollo tecnológico y el PC se retroalimentan para mejorar los resultados mutuos; y (7) el PC promueve la innovación fuera de los contextos en los que se aplica en primera instancia. No en vano, de estas potencialidades, comúnmente aceptadas, se deriva que en sus diferentes aproximaciones educativas (PC propiamente dicho, o robótica y programación) sean muchos los sistemas educativos que hayan apostado decididamente por incluirlo en el currículum, como es el caso de Canadá, Reino Unido, Finlandia, Australia, etc. (Acevedo Borrega, 2016; Bocconi, Chioccariello, Dettori, Ferrari, \& Engelhardt, 2016).

En su operativa, sin embargo, no existe tanto consenso sobre cuáles son sus elementos o componentes definitorios. Así, por ejemplo, podemos hablar de elementos como la abstracción, la generalización, la descomposición, el pensamiento algorítmico y la depuración (Angeli et al., 2016), a los que se podrían añadir la automatización (Bocconi et al., 2016), la recogida y el procesamiento de información y la 
representación (Barr \& Stephenson, 2011), los análisis ascendente y descendente, la heurística, el pensamiento divergente, la recursividad, la iteración, la aproximación y la metacognición (Zapata-Ros, 2015), todo ello como elementos intrínsecos. Y también elementos actitudinales implícitos como la seguridad, la perseverancia o la colaboración (Bocconi et al., 2016), que en buena medida entroncan con el currículum transversal o nuclear.

Si focalizamos en las primeras características, insinuadas por Wing $(2006,2010)$, y desarrolladas por Angeli (2016), es fácil comprender por qué el desarrollo del PC en el sistema educativo ha gozado de especial predicamento ligado a planteamientos de robótica o de programación (Furber, 2012; Yadav, Gretter, Good, \& Mclean, 2017; Yadav, Hong, \& Stephenson, 2016). De hecho, esta relación del PC con la robótica especialmente es intensa fuera del ámbito educativo formal. Con todo, la relación entre el PC y la robótica no es biunívoca, en el sentido de que la robótica no necesariamente garantiza el desarrollo del PC, ni el PC puede adquirirse únicamente por medio de la robótica (Bocconi, Chioccariello, Dettori, Ferrari, Engelhardt, et al., 2016a; Voogt, Fisser, Good, Mishra, \& Yadav, 2015; Yadav et al., 2017). Y, de igual modo ocurre con la competencia digital, que puede ir íntimamente ligada al desarrollo del PC, especialmente si focalizamos en los elementos que añaden Barr y Stephenson (2011) y ZapataRos (2015), pero que no necesariamente tiene por qué incidir en el PC (del mismo modo que se puede adquirir el PC sin que la competencia digital se vea beneficiada). En definitiva, aunque los lenguajes de programación puramente computacionales y las tecnologías puedan ayudarnos en la adquisición del PC, esta no es automática ni tiene por qué darse implícitamente, sino que requiere de acciones específicas. Además, y como consecuencia de esto, si bien es común pensar que el PC puede constituir una materia específica en el currículum ( $y$, de hecho, así ha sido en muchos contextos educativos nacionales que hemos mencionado antes) (Brennan \& Resnick, 2012), también hay quienes abogan por su mayor efectividad cuando se integra en el resto del currículum, como una estrategia de aprendizaje y no como una materia finalista (Bocconi, Chioccariello, Dettori, Ferrari, Engelhardt, et al., 2016b; Morreale, Jimenez, Goski, \& Stewart-Gardiner, 2012).

La práctica, sin embargo, parece ir por otro lado, y se asume común y acaso erróneamente que solo con plantear situaciones de aprendizaje en que intervenga la programación o la robótica se adquiere el PC (Yadav et al., 2017). Por ello, en los últimos años surgen demandas por plantear realmente cuáles son -o deberían ser- las mejores metodologías que nos permitan asegurar que el alumnado mejora su nivel de PC (Adell Segura, Esteve-Mon, Llopis Nebot, \& Valdeolivas Novella, 2017), y que tiene su máxima expresión en las recomendaciones del INTEF para el contexto español (Bocconi et al., 2016), que abogan por una integración multidisciplinar del PC más allá de planteamientos restrictivos como los habitualmente ligados a la programación estricta. O, más en el contexto internacional, que cuajan en modelos pedagógicos como el CTPF (por las siglas inglesas Computational Thinking Pedagogical Framework) (Kotsopoulos et al., 2017), que propone concretar la utilización de la programación (del 
coding puro) a fases determinadas de una secuencia, que empezaría por defecto sin elementos tecnológicos: una primera fase de programación analógica (unplugged), una de experimentación poco guiada y más bien lúdica (tinkering), una tercera de experimentación pautada, bajo la forma de proyectos (making) y una última integradora (remixing). Indudablemente, solo se trata de un modelo, por lo que es importante continuar con la investigación para tener claro cuál es la mejor secuencia de aprendizaje del PC (Adell Segura et al., 2017; Grover \& Pea, 2013).

Si la adquisición del PC por parte del alumnado ni es espontánea ni queda garantizada por su participación en actividades de robótica o programación sin más, es obvio que los docentes deben planificarlas según unos principios determinados. Y eso nos lleva de modo ineludible a su formación al respecto. Por un lado, la literatura indica que, en efecto, se han realizado no pocos esfuerzos en ese sentido (Morreale et al., 2012; Morreale \& Joiner, 2011); sin embargo, en líneas generales la capacitación del profesorado en activo ha sido de tipo instrumental (en la propia destreza de la programación) (Bustillo \& Garaizar, 2015); y, como resultado de ello, el impacto que ha tenido en la implementación de estrategias de PC en las aulas de educación obligatoria es bastante limitado.

Que hay que formar a los docentes es un lugar común que goza de consenso general. Los esfuerzos se dirigen últimamente tanto hacia la propia formación de los docentes tanto como en los modelos pedagógicos asociados al PC (Morreale et al., 2012). Muchos docentes aplican ya estas estrategias sin tener conciencia de ello (Barr \& Stephenson, 2011; Yadav et al., 2017), si bien no es algo que deba presuponerse.

En cuanto a la formación, basta argüir que los propios docentes no han sido formados en PC y que sus experiencias en robótica o programación en sus respectivas formaciones iniciales ha sido escasa o nula (Bustillo Bayón, 2015); por ello, si pensamos que la primera condición es que el propio docente haya adquirido las mínimas destrezas técnicas como usuario (Adell Segura et al., 2017), es fácil colegir que un primer nivel de formación deba garantizar esa adquisición del dominio instrumental de las herramientas o de los lenguajes que quieran utilizarse. Así pues, es preciso planificar la formación del profesorado para que no solo sea competente desde la perspectiva del PC, sino también como diseñador e implementador de estrategias que ayudan a adquirirlo (Prieto-Rodriguez \& Berretta, 2014).

En paralelo están surgiendo modelos de formación en didáctica del PC, que en líneas generales intentan adaptar al ámbito concreto que nos ocupa el modelo TPACK (Mishra \& Koehler, 2006, 2008). Estos modelos indican de forma sintética que cualquier estrategia de formación orientada a los docentes deberá garantizar (1) que el docente identifica con claridad qué proyectos pueden dar cabida a situaciones de aprendizaje del PC; (2) que es capaz de identificar qué tecnologías son las más adecuadas a dichos proyectos; y (3) que identifica cuáles son las oportunidades de transformar la simbiosis entre dichos proyectos y dichas tecnologías en aprendizaje del PC. Aunque aún está por 
desarrollar y operativizar, es indudable que ofrece un marco conceptual dentro del cual es evidente que maestros generalistas o profesores de computación deben ser formados en destrezas específicas que garanticen su propia competencia como docentes de PC.

Hasta ahora se ha puesto el acento en la formación del profesorado en activo, como decíamos, con especial atención a la parte más técnica de su capacitación (Bustillo Bayón, 2015; Morreale et al., 2012; Yadav et al., 2017); por contra, se ha dejado desatendido el colectivo de los pre-service teachers, de modo que es poco frecuente encontrar que el PC se integra dentro de los módulos de formación de las facultades de Educación (Yadav et al., 2017). Sin embargo, las pocas experiencias de que se dispone evidencian que muy pocos esfuerzos (o, lo que es lo mismo, acciones puntuales) dan resultados muy interesantes. Así, por ejemplo, Bustillo (2015) señala que el cambio actitudinal es casi una constante en cualesquiera de las experiencias de formación con profesorado (in-service y pre-service), y también es común constatar que la mayoría de las intervenciones, incluso las más superficiales, ayudan a esclarecer el concepto de PC y a combatir los errores en su interpretación (Morreale \& Joiner, 2011). Y, por hablar de su impacto, aunque la literatura no es aún pródiga al respecto por la juventud del campo de conocimiento, se conviene que el profesorado participante rápidamente mejora su propia competencia en PC, así como en el diseño de estrategias de aprendizaje del PC (Morreale et al., 2012), que el intercambio de buenas prácticas suele ser productiva como estímulo al salto a la práctica (Ertmer \& Ottenbreit-Leftwich, 2010) y que la mayoría del profesorado que participa en sesiones de formación que van más allá de lo puramente instrumental acaba aplicando estrategias de PC en su propia práctica docente, aunque sea de modo superficial (Dapozo et al., 2016).

En nuestro contexto, como en muchos otros del mundo occidental, y como consecuencia de todo lo anterior, hay cada vez más escuelas e iniciativas educativas relacionadas con la robótica educativa y con la programación informática: por ejemplo, son habituales las propuestas que parten del uso de los kits educativos Lego-Mindstorms o Lego We-DO, de los robots infantiles The Bee-Bot, de la plataforma de programación Scratch o su versión infantil ScratchJR, o de los paneles de microcontroladores Arduino, entre otros (Estebanell Minguell, González Martínez, Peracaula Bosch, \& López Simó, 2017). Y, como decíamos antes, se han lanzado varias iniciativas para fomentar el PC a nivel local y nacional: campus, clubes de códigos, museos, proyectos y redes europeas, etc. En ese sentido, son interesantes los alcances que se han llevado a cabo en relación con el estudio de la introducción del PC en los estudios universitarios (García-Peñalvo, 2016; García-Peñalvo, Reimann, Tuul, Rees, \& Jormanainen, 2016) dentro de iniciativas Erasmus+ de ámbito europeo; o los avances en la conceptualización de las relaciones entre robótica y PC, también en el ámbito universitario (Fernández-Llamas, Conde, Rodríguez-Lera, Rodríguez-Sedano, \& García, 2018; Fernández-Llamas, Conde, Rodríguez-Sedano, Rodríguez-Lera, \& Matellán-Olivera, 2017) 
Sin embargo, volviendo al objeto de nuestra atención ahora, los maestros en formación, para lograr que realmente todo ello resulte exitoso en términos de adquisición del PC, el primer paso que hay que hacer es lograr que los docentes asimilen este cambio de mentalidad y lo incorporen en su práctica educativa (en su trabajo en el aula), y que los futuros maestros lo exploren durante su formación para que puedan aplicarlo en su futuro profesional en las escuelas. Ahora bien, ¿qué entienden nuestros futuros maestros por PC? ¿Realmente conocen qué implica? ¿Son capaces de distinguir el PC de la robótica o de la programación? ¿Qué objetivos creen que persigue el PC? Y, desde nuestra misión de formadores de formadores, ¿podemos ayudarles a cambiar sus prejuicios al respecto y a comprender mejor qué es el PC por medio de propuestas de formación integradas en los planes de estudio de las facultades de Educación?

Como primera fase del proyecto de investigación PECOFIM (El Pensament Computacional a la Formació de Mestres, https://pecofim.wixsite.com/pecofim), esta investigación intenta abordar estas cuestiones. Y, más en concreto, este artículo ofrece un análisis del concepto inicial y de las actitudes sobre el TC de los profesores en formación a partir de una encuesta, así como de cuál es su evolución tras la integración de un módulo sobre PC en la formación inicial de maestros.

Para ello, a continuación, ofrecemos un apuntemetodológico sobre las técnicas utilizadas, presentamos los datos recabados por medio del instrumento utilizado, así como su análisis, y retomamos la reflexión inicial para valorarla a la luz de esta interpretación y alcanzar nuestras conclusiones.

\section{Metodología}

La presente investigación es un análisis descriptivo con datos cualitativos y cuantitativos realizado durante la primavera de 2017 a través de un cuestionario creado específicamente para esta experiencia de innovación. La elección de esta técnica se fundamenta dentro de la estrategia global del proyecto de innovación, bajo el modelo de investigación basada en el diseño (Plomp \& Nieveen, 2010). Dentro de las aproximaciones iniciales, se consideró que un cuestionario como el utilizado podría satisfacer de forma eficaz las necesidades de información contextual para el análisis inicial de la experiencia. A partir de estas consideraciones iniciales, esta encuesta trató de explorar las siguientes preguntas:

- ¿Qué saben los maestros en formación sobre el PC antes de ser entrenados en él? ¿Qué acaban sabiendo después de participar en el módulo de PC?

- ¿Cuáles son sus actitudes y expectativas sobre el PC?

La muestra quedó constituida por un total de 163 estudiantes de los grados de Educación Primaria e Infantil de la Universitat de Girona (España) que respondieron a la encuesta, con el siguiente perfil 
resumido: el 14\% eran hombres, el 86 \% eran mujeres; el 29,4 \% dijo haber participado en experiencias anteriores relacionadas con la robótica y la programación, y el 70,6 \% dijo que no tenía experiencia previa. Como decíamos al inicio, la muestra quedó constituida en relación con el foco principal del proyecto de innovación, los futuros maestros, y como complemento a lo que se conoce ya sobre los maestros en activo y lo que pueda complementarse sobre ellos en sucesivas investigaciones.

Los datos cualitativos se codificaron a partir de un libro de códigos de categoría ad hoc creado después del análisis exploratorio; los datos cuantitativos fueron depurados, tabulados y analizados con estadísticas descriptivas básicas (test ANOVA para los grados de significación cuando se trabajó con el conjunto de la muestra, que reveló una distribución normal; test chi-cuadrado para los grados de significación cuando se trabajó con fracciones de la muestra cuya distribución no reunió condiciones de normalidad).

En relación con la experiencia de innovación, todos los alumnos tuvieron contacto, como mínimo, con una experiencia de formación general sobre el PC (concepto y posibles aplicaciones en el aula); además de esta sesión general, un 28,8 \% del alumnado participó en un módulo completo de seis semanas dedicado al PC, de 1,5 horas semanales, en los que se profundizó en el concepto y, por medio de una estrategia didáctica de PBL (por las siglas inglesas Problem Based Learning), tuvo que desarrollar secuencias de aprendizaje del PC para alumnado de Infantil y Primaria en un contexto lúdico, que se revisaron, se implementaron y se evaluaron. Todo este proceso de diseño instruccional fue aprovechado para reflexionar en torno al PC y a sus potencialidades educativas.

\section{Resultados}

\section{1. ¿Qué saben los maestros en formación sobre PC?}

Como podemos ver en la Tabla 1, antes de la formación en PC los maestros en formación conocen sólo parcialmente el concepto de PC. Pocos de ellos (3,4 \%) son capaces de definirlo correctamente cuando se lo pedimos. Por el contrario, la mayoría de ellos (69\%) produce definiciones más o menos cercanas a lo que entendemos por PC. Y casi un tercio de los informantes no sabe qué es el PC ni por aproximación, ya lo reconozcan explícitamente o no (cuando producen respuestas que no tienen nada que ver con el concepto). En cambio, después de la experiencia de formación (leve o intensa según hemos explicado en el epígrafe anterior), el porcentaje de alumnado capaz de definir apropiadamente el PC es sensiblemente mayor; y, por el contrario, desciende a la mitad la parte de la muestra incapaz de hacerlo. Aplicados los tests de varianza correspondientes, la diferencia entre las dos muestras es significativa al nivel de 0,00, con lo cual podemos concluir que la formación ha sido eficaz, al menos, en este sentido. Por tanto, podemos concluir que, en un primer nivel teórico, la experiencia de 
innovación, por medio de la exposición de los participantes al concepto de PC, les ayuda a mejorar su conocimiento sobre él.

\begin{tabular}{|l|c|c|}
\hline & $\begin{array}{c}\text { Antes } \\
(\%)\end{array}$ & $\begin{array}{c}\text { Después } \\
(\%)\end{array}$ \\
\hline Lo conocen & 3,4 & 38,3 \\
\hline Lo conocen parcialmente & 69 & 48,9 \\
\hline No lo conocen & 16,4 & 12,8 \\
\hline Dicen que no lo conocen & 11,2 & 0 \\
\hline
\end{tabular}

Tabla 1. ¿Saben qué es el PC?

En la Tabla 2, podemos ver cómo se organizan las diferentes clases de respuestas ofrecidas por los estudiantes cuando se les pide que formulen sus definiciones de PC antes de cualquier formación al respecto. Muchos de ellos lo relacionan con la capacidad de resolver problemas en general $(21,4 \%)$ o con el uso de tecnología (17,3\%). Y otro importante grupo de informantes lo asocia con procesos de programación de dispositivos informáticos (22,4\%). El resto de informantes ofrece definiciones relacionadas con temas educativos en general (1\%), gestión de la información (2.6\%) u otros enfoques más generales (4.1\%). Como podemos ver, pocos están muy cerca del concepto de PC real $(4,1 \%)$. En cambio, los porcentajes de respuesta tras la formación en PC, en consonancia con los datos de la Tabla 1, son mucho más cercanos al concepto real de PC. Además, por un lado mejorar las adhesiones a tópicos más relacionados con el PC, mientras que descienden aquellas de tipo más genérico (como resolución de problemas).

\begin{tabular}{|l|c|c|}
\hline & $\begin{array}{c}\text { Before } \\
(\%)\end{array}$ & $\begin{array}{c}\text { After } \\
(\%)\end{array}$ \\
\hline Programación & 22,4 & 25,5 \\
\hline Educación, en general & 1 & 4,3 \\
\hline Resolución de problemas & 21,4 & 10,6 \\
\hline Resolución de problemas con TIC & 17,3 & 2,1 \\
\hline Pensamiento computacional & 4,1 & 38,1 \\
\hline Definiciones imprecisas & 6,1 & 6,4 \\
\hline Gestión de la información & 2,6 & 2,1 \\
\hline
\end{tabular}

Tabla 2. ¿Qué piensan que es el PC? ¿Con qué lo relacionan?

Otra de las cuestiones que nos podemos plantear en este punto tiene que ver con la relación que puede establecerse entre el conocimiento que los futuros maestros tienen del concepto de PC y su experiencia previa en actividades ligadas a la robótica o a la programación. En ese sentido, como vemos en la Tabla 3, se establece una correlación significativa (sig. $=0,016 ; p>0.05)$ y negativa $(-0.189)$ entre su conocimiento del concepto y su experiencia previa, lo que quiere decir que su participación en actividades relacionadas con la robótica o la programación a lo largo de su educación formal o 
no formal no solo les permite tener un mejor conocimiento previo, sino lo contrario. Hasta cierto punto, parte de ello puede deberse al sesgo que introduce el propio hecho de que los alumnos, en general, hayan participado en experiencias educativas que pertenecen específicamente al ámbito de la robótica y de la programación, y no enfocadas en general al PC, menos específico y más transversal. Por ello, esta experiencia previa les lleva a pasar el concepto de PC por el cedazo de lo conocido, y no tanto de aquello que realmente podríamos asumir que es.

\begin{tabular}{|c|c|c|}
\hline & & $\begin{array}{c}\text { Experiencia } \\
\text { previa }\end{array}$ \\
\hline Conocimiento Concepto PC & Cor. Pearson & -0.189 \\
\cline { 2 - 3 } & Sig. (bilateral) & 0.016 \\
\hline
\end{tabular}

Tabla 3. Correlación entre conocimiento del concepto de PC y experiencia previa

\section{2. ¿Qué elementos del PC reconocen como tales?}

En esta segunda sección, después de pedirles que intentaran definir el PC, preguntamos a nuestros maestros en formación qué elementos consideraban que formaban parte del concepto. Se ofreció una lista de nueve elementos, incluyendo tres elementos relacionados con el PC (segmentación de tareas, toma de decisiones y testeo), tres más relacionados con la competencia digital (gestión de la información, comunicación y gestión de los riesgos) y los últimos tres relacionados con otras competencias transversales (reglas, autonomía y coordinación). Aunque el elemento que más generalmente considera parte del PC, la toma de decisiones, de hecho, es uno de ellos, en general parece que no está claro para nuestros docentes en formación qué elementos son específicos de la TC y cuáles no. En efecto, se puede ver que la gestión de la información y los riesgos asociados con la red son elementos que consideran parte del concepto $\mathrm{CT}$, mientras que están preferentemente asociados con la competencia digital. Y, por otra parte, el testeo, uno de los rasgos asociados a nuestro concepto, apenas se identifica de esa manera por la mitad de la muestra. Todas estas preguntas se pueden ver en la Figura 1. En líneas generales, todo ello apunta en la misma línea de lo anterior, pues esta dificultad en la identificación de los elementos característicos del PC no deja de ser otra cara de esa falta de conocimiento de la que hablábamos antes (característica incluso en aquellos alumnos que sí que reconocían tener experiencia previa en robótica o en programación). 


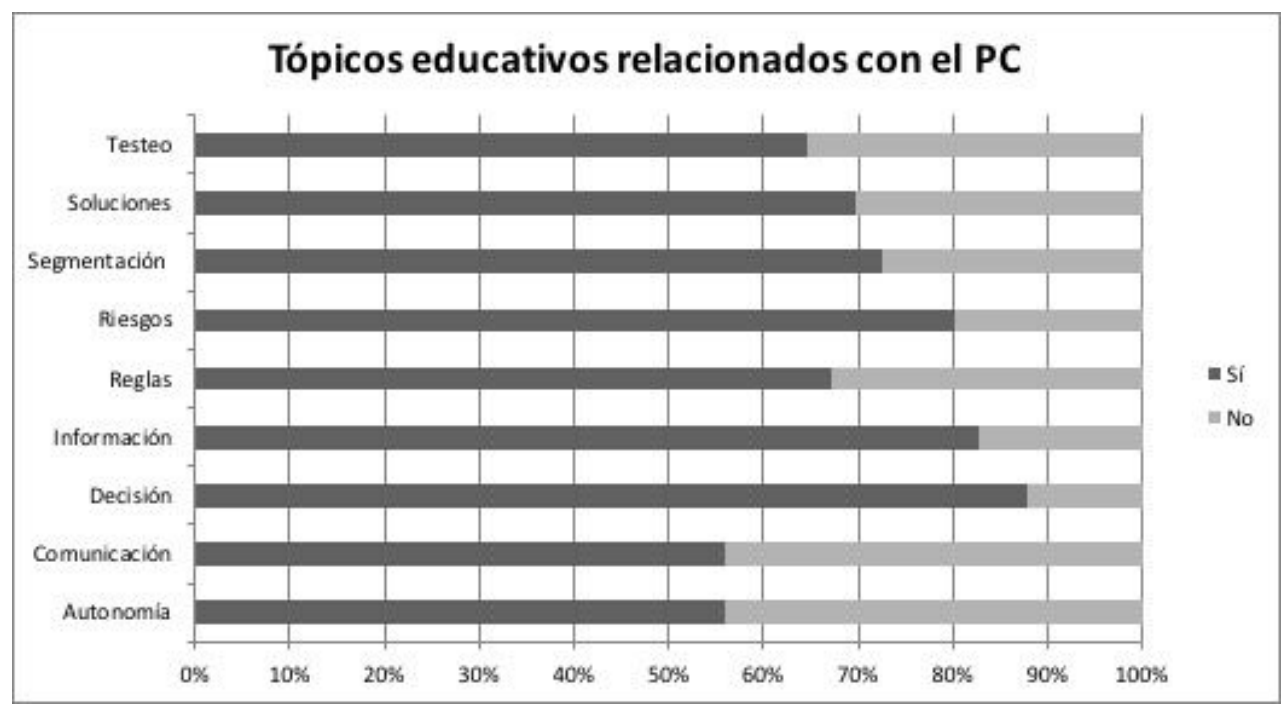

Figura 1. Tópicos educativos relacionados con el PC

\section{3. ¿Cuáles son sus actitudes y expectativas sobre PC?}

Por otro lado, como podemos ver en la Figura 2, nos interesamos por las actitudes y por las expectativas de los docentes en formación en relación con el PC, y por eso les preguntamos si se sentían bien formados en este sentido, si el PC les parecía interesante, cuál era su propia capacidad como maestros para incluir el PC en su práctica educativa, si se sentían seguros para diseñar actividades de PC y, finalmente, si el PC les parecía relevante desde un punto de vista educativo. En general, las actitudes y las expectativas de nuestra muestra son positivas, considerando que dicen que el PC es relevante e interesante. Sin embargo, como se esperaba, no se sienten muy entusiasmados con su propia capacidad como futuros maestros para diseñar e implementar experiencias de aprendizaje vinculadas al PC. Y, como podemos ver también, esto tiene mucho que ver con la idea de que, en general, se sienten poco formados al respecto.

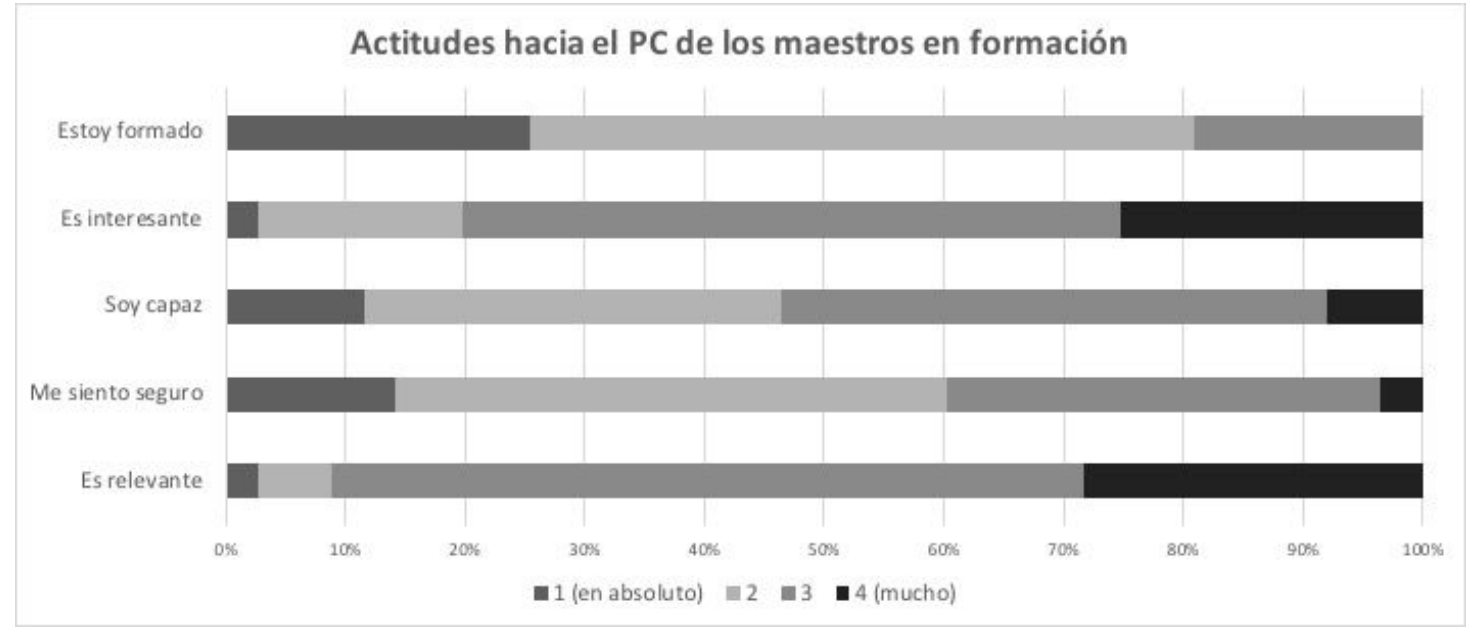

Figura 2. Actitudes hacia el PC de los profesores en formación 
A partir de aquí, podemos plantearnos cuáles son las relaciones que se establecen entre estos componentes actitudinales. Para ello, si atendemos a las correlaciones que se establecen entre estos elementos, vemos que son positivas y significativas en todas aquellos elementos que se relacionan con su percepción de capacidad para implementar estrategias didácticas ligadas al PC, excepto con la relevancia que consideren que tiene. Así pues, se sienten más capaces de aplicarlas al aula en la medida en que consideran que el PC es interesante, se sienten seguros para llevarlo a cabo y perciben que tienen la formación adecuada para ello. Lo que se comprueba en la Tabla 4, en la que destacamos en cursiva los valores que comentamos para facilitar la lectura.

\begin{tabular}{|c|l|l|c|c|c|c|}
\hline & & relevancia & seguridad & capacidad & interés & formación \\
\hline \multirow{2}{*}{ relevancia } & Cor. Pearson & & 0.008 & -0.007 & 0.018 & -0.004 \\
\cline { 2 - 7 } & Sig. (bilateral) & & 0.916 & 0.932 & 0.825 & 0.961 \\
\hline \multirow{2}{*}{ seguridad } & Cor. Pearson & 0.008 & & 0.995 & 0.994 & 0.997 \\
\cline { 2 - 7 } & Sig. (bilateral) & 0.916 & & 0.000 & 0.000 & 0.000 \\
\hline \multirow{2}{*}{ capacidad } & Cor. Pearson & 0.007 & 0.995 & & 0.993 & 0.996 \\
\cline { 2 - 7 } & Sig. (bilateral) & 0.932 & 0.000 & & 0.000 & 0.000 \\
\hline \multirow{2}{*}{ interés } & Cor. Pearson & 0.018 & 0.994 & 0.993 & & 0.993 \\
\cline { 2 - 7 } & Sig. (bilateral) & 0.825 & 0.000 & 0.000 & & 0.000 \\
\hline \multirow{2}{*}{ formación } & Cor. Pearson & 0.004 & 0.997 & 0.996 & 0.993 & \\
\cline { 2 - 7 } & Sig. (bilateral) & 0.961 & 0.000 & 0.000 & 0.000 & \\
\hline
\end{tabular}

Tabla 4. Correlación entre elementos actitudinales

Podemos dar un paso más allá y ver qué orientación tendría un modelo predictivo construido a partir de este diagnóstico inicial de las correlaciones. Para ello, una regresión múltiple como la que se recoge en la Tabla 5, a continuación, nos ofrece una $\mathrm{R}=0.997$, con lo que el modelo nos permitiría explicar 9273.33 de los 9336.84 puntos que tiene el modelo, lo cual es altamente positivo. En dicho modelo, es la formación el predictor que más información nos aporta, seguido por el interés y por la seguridad; todo ello nos hace pensar que cuando mejor formados perciba el alumnado que está más capaz se sentirá de llevar a cabo en el aula actividades relacionadas con el PC.

\begin{tabular}{|c|l|c|c|c|}
\hline \multicolumn{2}{|c|}{} & $\begin{array}{c}\text { Coeficientes } \\
\text { tipificados }\end{array}$ & & Sig. \\
\cline { 3 - 5 } \multicolumn{2}{|c|}{} & Beta & & \\
\hline \multirow{3}{*}{1} & (Constante) & & 2,786 &, 006 \\
\cline { 2 - 5 } & Seguridad &, 269 & 2,954 &, 004 \\
\cline { 2 - 5 } & Interés &, 207 & 3,231 &, 002 \\
\cline { 2 - 5 } & Formación &, 523 & 6,223 &, 000 \\
\hline
\end{tabular}




\section{Conclusiones}

Como decíamos al inicio, una parte de esta investigación partía de la idea de que es preciso formar a los docentes comenzando por el propio concepto de PC, si queremos que este se integre en el currículum, sea bajo la forma que sea, de forma productiva. En el fondo, yacía la idea de que en líneas generales los maestros en formación continúan permaneciendo ajenos al PC en sus planes de estudio, lo que dificulta que puedan ser competentes al respecto (Yadav et al., 2017); y que, incluso en aquellos casos en que los maestros han tenido experiencias ligadas a la robótica o formación específica en programación educativa, han podido adquirir únicamente competencias instrumentales que difícilmente trasvasan a la práctica productiva (Bustillo Bayón, 2015).

La experiencia llevada a cabo, en este sentido, confirma dos de las grandes ideas que subyacen a la revisión bibliográfica inicial: (1) los futuros maestros, incluso aunque hayan participado como alumnos en actividades de robótica o programación, desconocen en líneas generales qué es el PC (cabría pensar, a tenor de los datos recabados, incluso que haber participado en estas experiencias antes les resta que les suma en este sentido); y (2) su participación en una experiencia formativa integrada en el plan de estudio universitario, incluso aunque resulte superficial, les permite mejorar el concepto de PC (Morreale \& Joiner, 2011) y romper las asociaciones biunívocas erróneas entre PC y competencia digital y programación o robótica (Bocconi et al., 2016b; Voogt et al., 2015). Además, el análisis llevado a cabo también nos permite confirmar que, en frío, nuestros futuros maestros tienen buenas expectativas hacia el PC y hacia su aplicación en el aula de Primaria, en consonancia con lo que indica los estudios (Bustillo \& Garaizar, 2015; Bustillo Bayón, 2015), y esto es especialmente interesante en la medida en que se sienten inseguros para hacerlo (por sus carencias formativas específicas), e incluso a pesar de las lagunas que tengan al respecto de qué es exactamente el PC y cuáles son sus potencialidades educativas. Ello, a buen seguro, es una consecuencia indirecta de este ambiente de opinión que recientemente documentamos, en virtud del cual el propio PC se ha convertido en tema de actualidad (Estebanell Minguell et al., 2017; European Commission/EACEA/ Eurydice, 2012; Fluck et al., 2016).

Las conclusiones que se derivan de este diagnóstico son importantes por cuanto marcan la pauta de lo que debe ser la formación de maestros, si queremos trabajar por esa integración efectiva y productiva del PC en las aulas, que debe empezar forzosamente por reflexionar acerca del propio concepto de PC y de sus potencialidades. Es imprescindible ofrecer a los futuros maestros (y también a los maestros en ejercicio) oportunidades de desarrollo profesional ligadas a su formación en PC (Yadav et al., 2016), que se centren especialmente en la dimensión pedagógica y que trasciendan la puramente instrumental (Bustillo Bayón, 2015), pues es la dimensión que permite que el PC se alíe con 
la creatividad, con la valentía y con la competencia docente general de los docentes al servicio de la innovación educativa. En ese sentido, los talleres sobre PC son una herramienta fantástica en primera instancia, pues permiten la mejora del concepto de PC de los asistentes y mejoran sus actitudes al respecto (Morreale et al., 2012). Les permiten asumir un concepto más claro y operativo del PC (Angeli \& Valanides, 2005; European Commission/EACEA/Eurydice, 2012; Fluck et al., 2016; Wing, 2006), lejos de los clichés estrictos de programación o robótica a los que habitualmente se asocia. Y qué duda cabe de que, además, les dota de mejores estrategias como individuos frente a la resolución de sus problemas personales, profesionales y académicos. Con todo y con eso, si se quiere que la formación tenga un verdadero impacto, es preciso abordarla de forma profunda, integral y planificada (Adell Segura et al., 2017; Yadav et al., 2017) a lo largo de todos los estudios universitarios de maestro.

Llegados a este punto, podemos resumir brevemente nuestros próximos pasos en este ámbito de investigación, así como las nuevas líneas que se abren a partir de este primer análisis. Por un lado, en relación con el proyecto en que este análisis se circunscribe, los siguientes pasos van encaminados a medir y calibrar el alcance de las experiencias formativas con fututos maestros en relación con el PC. En definitiva, que conozcan el concepto de PC, que identifiquen sus elementos y que sus actitudes hacia él sean positivas solo es la primera parte de un camino que al final debe dotarles de las estrategias necesarias para diseñar experiencias de aprendizaje que animen a la introducción del PC en Educación Primaria. Queda, pues, seguir evaluando el proyecto de innovación para ver la calidad de esos diseños por parte de los futuros maestros. Por otro lado, y en relación con la identificación entre robótica, programación y PC, esta investigación inicial evidencia la necesidad de seguir ahondando en la investigación de las relaciones entre todos esos elementos, tan en boga en el ámbito de conocimiento, y en relación con la oposición entre los llamados learn to code o code to learn.

\section{Agradecimientos}

El Proyecto PECOFIM (El Pensament Computacional a la Formació Inicial de Mestres), cuyo identificador es 2015 ARMIF 00031, ha sido financiado en la convocatoria de ayudas ARMIF 2015 para la Mejora de la Formación Inicial de Maestros de la Agència de Gestió d’Ajuts Universitaris (AGAUR) de la Generalitat de Catalunya (España).

\section{Referencias}

Acevedo Borrega, J. (2016). El pensamiento computacional en la educación obligatoria. Una revisión sistemática de la literatura. Universidad de Extremadura.

Adell Segura, J., Esteve-Mon, F. M., Llopis Nebot, M. Á., \& Valdeolivas Novella, M. G. (2017). El Pensamiento Computacional en la formación inicial del profesorado de Infantil y Primaria. In Actas 
de las XXV Jornadas Universitarias de Tecnología Educativa (pp. 1-7). Burgos: Red Universitaria de Tecnología Educativa.

Angeli, C., \& Valanides, N. (2005). Preservice elementary teachers as information and communication technology designers: An instructional systems design model based on an expanded view of. Journal of Computer Assisted Learning, 1(4), 292-302. doi:https://doi.org/10.1111/j.1365-2729.2005.00135.x

Angeli, C., Voogt, J., Fluck, A., Webb, M., Cox, M., Malyn-Smith, J., \& Zagami, J. (2016). A K-6 Computational Thinking Curriculum Framework: Implications for Teacher Knowledge. Educational Technology \& Society, 19(3), 47-57.

Barr, V., \& Stephenson, C. (2011). Bringing Computational thinking to K-12: What is involved and what is the role of the computer science education community? ACM Inroads, 2(1), 48-54. doi:https://doi. org/10.1145/1929887.1929905

Bocconi, S., Chioccariello, A., Dettori, G., Ferrari, A., \& Engelhardt, K. (2016). El Pensamiento Computacional en la Enseñanza Obligatoria (Computhink). Implicaciones para la política y la práctica. Developing Computational Thinking in Compulsory Education. Implications for policy and practice. https://doi.org/10.2791/792158

Bocconi, S., Chioccariello, A., Dettori, G., Ferrari, A., Engelhardt, K., Kampylis, P., \& Punie, Y. (2016a). Developing Computational Thinking : Approaches and Orientations in K-12 Education. In Proceedings of the EdMedia 2016 Conference (pp. 1-7). Vancouver, BC, Canada: EdMedia.

Bocconi, S., Chioccariello, A., Dettori, G., Ferrari, A., Engelhardt, K., Kampylis, P., \& Punie, Y. (2016b). Exploring the Field of Computational Thinking As a 21st Century Skill. In Proceedings of the EDULEARN16 (Vol. 16, pp. 4725-4733). Barcelona: EDULEARN. doi:https://doi.org/10.21125/edulearn.2016.2136

Brennan, K., \& Resnick, M. (2012). New frameworks for studying and assessing the development of computational thinking. In Annual American Educational Research Association meeting (pp. 1-25). Vancouver, BC, Canada. Retrieved from http://web.media.mit.edu/ kbrennan/files/Brennan_Resnick_ AERA2012_CT.pdf

Bustillo Bayón, J. (2015). Formación del profesorado con scratch: análisis de la escasa incidencia en el aula. Opción, 31(1), 164-182. Retrieved from http://www.redalyc.org/pdf/310/31043005010.pdf

Bustillo, J., \& Garaizar, P. (2015). Scratching the surface of digital literacy... but we need to go deeper. In Proceedings of the Frontiers in Education Conference (FIE). Madrid: IEEE. doi:https://doi.org/10.1109/ FIE.2014.7044224 
Cela-Ranilla, J., Esteve, V., Esteve Mon, F. M., González Martínez, J., \& Gisbert Cervera, M. (2017). El docente en la sociedad digital: una propuesta basada en la pedagogía transformativa y en la tecnología avanzada. Profesorado. Revista de Currículum y Formación del Profesorado, 21(1), 403-422.

Dapozo, G., Petris, R., Greiner, C., Espíndola, M. C., Company, A. M., \& López, M. (2016). Capacitación en programación para incorporar el pensamiento computacional en las escuelas. In A. E. De Giusti, I. Sattolo, J. S. Ierache, \& P. M. Pesado (Eds.), XI Congreso de Educación en Tecnología y Tecnología en Educación (pp. 113-121). Buenos Aires (Argentina): Universidad de Morón. Retrieved from http:// teyet-revista.info.unlp.edu.ar/wp-content/uploads/2016/08/Capacitación-en-programación-paraincorporar-el-pensamiento-computacional-en-las-escuelas.pdf

Ertmer, P. A., \& Ottenbreit-Leftwich, A. T. (2010). Teacher Technology Change: How Knowledge, Confidence, Beliefs, and Culture Intersect. Journal of Research on Technology in Education, 42(3), 1539-1523. doi:https://doi.org/10.1080/15391523.2010.10782551

Estebanell Minguell, M., González Martínez, J., Peracaula Bosch, M., \& López Simó, V. (2017). About the concept of Computational Thinking and its educational potentialities by pre-service teachers. In López Chova, L., López Martínez, A., \& Candel Torres, I. (Eds.), Proceedings of the 2017 EduLearn 9th International Conference on Education and New Learning Technologies (pp. 6624-6630). Barcelona: EDULEARN. doi:https://doi.org/10.21125/edulearn.2017.2510

European Commission/EACEA/Eurydice. (2012). Developing Key Competences at School in Europe: Challenges and Opportunities for Policy. Eurydice Report. Luxembourg: Publications Office of the European Union. doi:https://doi.org/10.2797/93204

Fernández-Llamas, C., Conde, M. A., Rodríguez-Lera, F. J., Rodríguez-Sedano, F. J., \& García, F. (2018). May I teach you? Students' behavior when lectured by robotic vs. human teachers. Computers in Human Behavior, 80, 460-469. doi:https://doi.org/10.1016/j.chb.2017.09.028

Fernández-Llamas, C., Conde, M. Á., Rodríguez-Sedano, F. J., Rodríguez-Lera, F. J., \& Matellán-Olivera, V. (2017). Analysing the Computational Competences Acquired by K-12 Students When Lectured by Robotic and Human Teachers. International Journal of Social Robotics. doi:https://doi.org/10.1007/ s12369-017-0440-9

Fluck, A., Webb, M., Cox, M., Angeli, C., Malyn-smith, J., Voogt, J., \& Zagami, J. (2016). Arguing for Computer Science in the School Curriculum. Educational Technology \& Society, 19(3), 38-46.

Furber, S. (2012). Shut down or restart? The way forward for computing in UK schools. London (UK): The Royal Society. 
García-Peñalvo, F. J. (2016). Presentation of the TACCLE3 Coding European Project. Salamanca. Retrieved from http://repositorio.grial.eu/handle/grial/654

García-Peñalvo, F. J., Reimann, D., Tuul, M., Rees, A. M., \& Jormanainen, I. (2016). TACCLE 3, O5: An overview of the most relevant literature on coding and computational thinking with emphasis on the relevant issues for teachers. Belgium. doi:https://doi.org/10.5281/zenodo.165123.

Grover, S., \& Pea, R. (2013). Computational Thinking in K-12: A Review of the State of the Field. Educational Researcher, 42(1), 38-43. doi:https://doi.org/10.3102/0013189X12463051

Kotsopoulos, D., Floyd, L., Khan, S., Namukasa, I. K., Somanath, S., Weber, J., \& Yiu, C. (2017). A Pedagogical Framework for Computational Thinking. Digital Experiences in Mathematics Education, 2017(March), 1-18. doi:https://doi.org/10.1007/s40751-017-0031-2

Mishra, P., \& Koehler, M. J. (2006). Technological Pedagogical Content Knowledge: A Framework for Teacher Knowledge. Teachers College Record, 108(6), 1017-1054. doi:https://doi.org/10.1111/j.14679620.2006.00684.x

Mishra, P., \& Koehler, M. J. (2008). Introducing technological pedagogical content knowledge. Paper presented at the Annual Meeting of the American Educational Research Association. New York City, March 24-28, 2008.

Morreale, P., \& Joiner, D. (2011). Changing Perceptions of Computer Science and Computational Thinking Among High School Teachers. Journal of Computing Sciences in Colleges, 26(6), 71-77.

Morreale, P., Jimenez, L., Goski, C., \& Stewart-Gardiner, C. (2012). Measuring the impact of computational thinking workshops on high school teachers. Journal of Computing Sciences in Colleges, 27(6), 151157.

Plomp, T., \& Nieveen, N. (2010). An Introduction to Educational Design Research. Enschede (Netherlands): SLO. Netherlands institute for curriculum development. Retrieved from http://www.slo. nl/downloads/2009/Introduction_20to_20education_20design_20research.pdf/download

Prieto-Rodriguez, E., \& Berretta, R. (2014). Digital Technology Teachers' Perceptions of Computer Science: It is no all about programming. In Frontiers in Education Conference (pp. 1-5). USA: IEEE.

Voogt, J., Fisser, P., Good, J., Mishra, P., \& Yadav, A. (2015). Computational thinking in compulsory education: Towards an agenda for research and practice. Education and Information Technologies, 20(4), 715-728. doi:https://doi.org/10.1007/s10639-015-9412-6

Wing, J. M. (2006). Computational Thinking. Communications of the ACM, 49(3), 33-35. doi:https:// doi.org/10.1145/1118178.1118215 
Wing, J. M. (2010). Computational Thinking: What and Why? The Link Magazine.

Yadav, A., Gretter, S., Good, J., \& Mclean, T. (2017). Computational Thinking in Teacher Education. In Spector, M., Bishop, M. J., \& Ifenthaler, D. (Eds.), Emerging Research, Practice, and Policy on Computational Thinking (pp. 205-220). Bloomington (IN, US): Springer. doi:https://doi.org/10.1007/978-3-319-526911

Yadav, A., Hong, H., \& Stephenson, C. (2016). Computational Thinking for All: Pedagogical Approaches to Embedding 21st Century Problem Solving in K-12 Classrooms. TechTrends, 60(6), 565-568. doi:https://doi.org/10.1007/s11528-016-0087-7

Zapata-Ros, M. (2015). Pensamiento computacional: Una nueva alfabetización digital. Revista de Educación a Distancia (RED), 46, 1-47. doi:https://doi.org/10.6018/red/46/4 